\section{Star observer}

\section{Bernard Lovell}

Edwin Hubble: Mariner of the Nebulae. By Gale E. Christianson. Farrar, Straus and Giroux: 1995. Pp. 420. \$27.50.

NowADAYs it would be difficult to hold any discussion about the Universe without reference to the Hubble constant, the Hubble time, the Hubble flow or, indeed, the Hubble telescope. No doubt Edwin Hubble would be content that the space telescope bore his name, but whether he would be pleased that his measurements on the redshift of the galaxies implies a Big Bang origin for the Universe is a different matter.

In this scholarly book, Gale Christianson reveals that Hubble was never toler-

nal to Shapley's Milky Way, would imply velocities far exceeding the velocity of light. In the two historic nights of 4 and 5 October 1923, Hubble recognized the Cepheid variable stars in the M31 spiral, and their period-luminosity curves left no room for doubt that the spiral was, indeed, an "island universe" lying far beyond the boundaries of Shapley's Milky Way.

It is fortunate that Shapley soon departed to Harvard, because he refused to believe the evidence for the Cepheids in M31. The depth of the rift between Hubble and Shapley is revealed by Christianson's references to Milton Humason, Hubble's faithful assistant. Humason maintained that before Shapley left Mount Wilson, he had shown him a plate of M31 on which he had marked the positions of Cepheids. Shapley refused to believe that they were Cepheids in M31, "then calmly took out his handkerchief, turned the plate over, and wiped them clean of Humason's marking".

With Humason's help, Hubble soon accumulated the data that established the linear relationship between redshift and distance of the nebulae (he always refused to use the word galaxies). In his 1929 and 1931 papers, Hubble published the velocity-distance relationship to 100 million light years, implying velocities of 20,000 $\mathrm{km} \mathrm{s}^{-1}$, but then, and ever afterwards, he was never convinced that these velocities implied an expansion of the Universe.

Initially, Hubble had good reason for this caution because the extrapolation to earlier cosmic times implied a timescale for the Universe less than the age of the Earth. With Hubble absent during the Second World War (he was at Maryland's Aberdeen Proving Grounds as head of external ballistics), Walter Baade was able to use the 100-inch telescope under blackout conditions. He discovered that there were two populations of the Cepheid variables and that a calibration error implied ences that so greatly dominated Hubble's subsequent career. Hubble was deeply that the Hubble distances and timescales had been underestimated by a factor of two. Although this removed the apparent conflict between the age of the Universe and that of the Earth, Hubble still would not concede that his measurements implied the large-scale expansion of the Universe.

Christianson gives an accurate account of Hubble's astronomical researches and of the lifestyle that formed the background to the astronomy. After the First World War, Hubble arrived at Mount Wilson as Major Hubble and to many, and particularly to his assistant Humason, he was always "the Major". He was an autocrat among scientists, insistent on formality and etiquette on the mountain and taking the liberty of making frequent and prolonged visits to England with his wife, Grace. These absences caused so much annoyance to Walter Adams, the director of the observatory, and to Vannevar Bush, president of the Carnegie Institution, that Hubble was not appointed as Adams's successor in 1945.

Hubble was stunned by the news that Ira S. Bowen was to be the new director, but the decision was a realistic one. The 200-inch telescope on Palomar Mountain was nearing completion and there is little evidence that Hubble would have been satisfactory as the director of the observatories. He was essentially an observational astronomer and, moreover, an astronomer with a single passionate interest in the nature and distribution of the nebulae. In 1949, in his first observations with the 200-inch telescope, he extended the redshift-distance relationship to a billion light years, but he remained unconvinced that his work had significance for any cosmological theory of the origin of the Universe. Christianson quotes from Hubble's manuscript notes in the Huntingdon Library about these observations that "there was no convincing evidence either of the expansion or non-expansion of the Universe".

Christianson's book is an important biographical work based on thorough searches of original material. In particular, the hundreds of pages of Grace Hubble's journals have revealed the extent of the Hubbles' social connections in England and the United States. The Hubbles relished the friendships that developed as Edwin's fame increased and, indeed, the book can be read with pleasure for its many vignettes on notable people of the time. Their life in the late 1930 s is epitomized by the remark by Grace, driving home in the moonlight after dining with Charlie Chaplin and his then wife, Paulette Goddard: "Now whom do we want to meet?"

Sir Bernard Lovell is at the Nuffield Radio Astronomy Laboratories, Jodrell Bank, Macclesfield, Cheshire SK11 9DL, UK. 